\title{
Enhanced radiation response in radioresistant MCF-7 cells by targeting peroxiredoxin II
}

\author{
This article was published in the following Dove Press journal: \\ Breast Cancer:Targets and Therapy \\ 10 October 2013 \\ Number of times this article has been viewed
}

\author{
Anthony Joseph Gomez \\ Diaz' \\ Daniel Tamae ${ }^{2}$ \\ Yun Yen ${ }^{3}$ \\ Jianjian $\mathrm{Li}^{4}$ \\ Tieli Wang' \\ 'Department of Chemistry and \\ Biochemistry, California State \\ University at Dominguez Hills, \\ Carson, CA, ${ }^{2}$ Center of Excellence \\ in Environmental Toxicology, \\ Department of Pharmacology, \\ University of Pennsylvania, \\ Philadelphia, PA, ${ }^{3}$ Department of \\ Clinical and Molecular Pharmacology, \\ Beckman Research Institute of City \\ of Hope National Medical Center, \\ Duarte, CA, ${ }^{4}$ Department of Radiation \\ Oncology, University of California \\ Davis, Sacramento, CA, USA
}

Correspondence: Tieli Wang Department of Chemistry and Biochemistry, California State University at Dominguez Hills, Carson, CA 90747, USA

Tel + I 3102433388

$\mathrm{Fax}+\mathrm{I} 3102432593$

Email twang@csudh.edu

\begin{abstract}
In our previous study, we identified that a protein target, peroxiredoxin II (PrxII), is overexpressed in radioresistant MCF+FIR3 breast-cancer cells and found that its expression and function is associated with breast-cancer radiation sensitivity or resistance. Small interference RNA (siRNA) targeting PrxII gene expression was able to sensitize MCF+FIR3 radioresistant breast-cancer cells to ionizing radiation. The major focus of this work was to investigate how the radiation response of MCF+FIR3 radioresistant cells was affected by the siRNA that inhibits PrxII gene expression. Our results, presented here, show that silencing PrxII gene expression increased cellular toxicity by altering cellular thiol status, inhibiting $\mathrm{Ca}^{2+}$ efflux from the cells, and perturbing the intracellular $\mathrm{Ca}^{2+}$ homeostasis. By combining radiotherapy and siRNA technology, we hope to develop new therapeutic strategies that may have potential to enhance the efficacy of chemotherapeutic agents due to this technology's property of targeting to specific cancer-related genes.
\end{abstract}

Keywords: siRNA, PrxII, radiation resistance, $\mathrm{Ca}^{2+}, \mathrm{MCF}+\mathrm{FIR} 3$

\section{Introduction}

Metastatic and recurrent breast cancer are distinct from early stage breast cancer. ${ }^{1}$ As a result, the recurrent form of the disease is resistant to existing targeted treatments, and the mortality rate is thus significantly higher, with as many as $40 \%$ relapsing with metastatic disease. ${ }^{1-3}$ The biological mechanisms underlying intrinsic and acquired resistance to chemotherapy and radiation therapy are therefore of considerable clinical significance. Studies have shown that breast-cancer cells that are able to propagate as mammospheres and possess cancer stem cell (CSC) properties are more radioresistant ${ }^{4}$ and the population of breast-cancer stem cells (BCSCs) is increased after chemotherapy. ${ }^{5}$ Therefore, the design of therapies targeting specific genes and proteins actively engaged in the physiopathology of radioresistant breast cancer is greatly demanded. Two-dimensional electrophoresis combined with mass spectrometry has been widely used to study protein expression profiles and to identify possible therapeutic targets. Using two-dimensional proteomic technology, we have identified a peroxiredoxin (Prx) II that plays an important role in the development of radioresistant breast-cancer cells that are enriched with a subpopulation of BCSCs. "PrxII" is a member of a ubiquitous family of antioxidant enzymes that normally respond to environmental oxidative stress or reactive oxygen species (ROS). ${ }^{7-9}$ Unchecked ROS can lead to oxidative damage in the cell, in turn leading to lipid peroxidation, protein modification, DNA modification, and strand breaks. Prxs play a central role in the cellular defense against oxidative damage through their peroxidase activity, whereby 
hydrogen peroxide, peroxynitrite, and a wide range of organic hydroperoxides are reduced and detoxified. ${ }^{10-12}$

The mechanism of detoxification by PrxII is the reduction of peroxides via cysteine residue in the active site. As a result of ionizing radiation (IR) or other environmental oxidative stress, ROS lead to the rapid inactivation of PrxII by the modification of the catalytic cysteine (R-SH) thiol to an inactive cysteine disulfide (R-S-S-R). This modification of the cysteine thiol to an inactive cysteine disulfide is completely reversible by the subsequent reduction of the inactive cysteine disulfide to regenerate the active PrxII cysteine. ${ }^{13}$ Evidence points to a pivotal role for PrxII in the cellular defense system. PrxII-overexpressing cells are more resistant to the environmental oxidative damage caused by inorganic hydrogen peroxide, organic t-butyl hydrogen peroxide, the heavy metal mercury, and anticancer drugs like cisplatin. ${ }^{14-17}$ Thus, PrxII is a promising candidate for genetargeted therapy. In our previous study, small interference RNA (siRNA) was transfected into the radioresistant breastcancer cells and was found to silence the gene expression of PrxII, resulting in enhanced radiosensitivity.

In the study reported here, we were interested in elucidating the mechanism by which inhibition by siRNA-PrxII of PrxII gene expression affects radiation-induced cell death in MCF+FIR3 radioresistant breast-cancer cells. Our hypothesis was that therapeutic IR combined with siRNA would enhance radiation-induced cell death by combining the gene-targeting property of siRNA and the cytotoxicity of radiation therapy. Our results showed that loss of protein thiol (PSH) and glutathione (GSH) (as a consequence of PrxII gene expression being silenced by PrxII-siRNA) is associated with increases in cellular toxicity, inhibition of $\mathrm{Ca}^{2+}$ efflux, and perturbation of $\mathrm{Ca}^{2+}$ homeostasis and results in enhanced radiosensitivity in MCF+FIR3 resistant cells. Thus, by combining radiotherapy with siRNA technology, we hope in future to be able to target cancer cells with specific genes involved in resistance. The results of these studies will be useful to help design advanced therapeutic strategies.

\section{Materials and methods Development of a radioresistant cell line}

MCF-7 human breast-cancer cells were purchased from the American Type Culture Collection (Manassas, VA, USA). The MCF+FIR30 cell line was developed as described in a previous publication. ${ }^{6} \mathrm{~A}$ radiation-resistant clone (MCF+FIR3) and a radiation-sensitive clone (MCF+FIS4) isolated from the MCF+FIR30 clonal populations were used for this study.

\section{Gene targeting by siRNA}

siRNA capable of targeting messenger RNAs (mRNAs) encoding PrxII was transfected using the same protocol as described before. ${ }^{6}$ The sequences of five PrxII-siRNA sense strands were:

5'-AGGTGAAGCTGTCGGACTA-3'
5'-GTGAGAGGACTTCCGCAA-3'
5'-GGAAGTACGTGGTCCTCTT -3'
5'-TCAAAGAGGTGAAGCTGTC-3'
5'-CGGACTACAAAGGGAAGTA-3'.

The sequence of the control siRNA was $5^{\prime}$-UCACUCCUAUAUCUUCUGUTT- ${ }^{\prime}$. This was used as a control for the PrxII gene-targeting experiments.

\section{Immunoblot analysis}

Immunoblot analysis was carried out using the standard protocol as described previously. ${ }^{18}$ PrxI, -II, and -III antibodies were used for these experiments. The immunoblot assays ran under the nonreducing experiments were performed in the absence of dithiothreitol (DTT) reducing reagent. Total protein concentration from the cell lysate was determined by Pierce ${ }^{\mathrm{TM}} \mathrm{BCA}$ assay kit (Thermo Fisher Scientific, Waltham, MA, USA). Scanned images were quantified by Kodak 1D Image Analysis software, (Kodak, Rochester, NY, USA), standardized by $\beta$-actin. Microsoft ${ }^{\circledR}$ Excel 2007 software (Microsoft Corporation, Redmond, WA, USA) was used for data analysis.

\section{3-[4,5-dimethylthiazol-2-yl]-2,5-diphenyl tetrazolium bromide (MTT) toxicity assay}

The MTT cytotoxicity method was used for cell viability measurements in multiwall plate format. Each experiment included a blank containing all of the reagents in a well without cells. MTT was dissolved in Dulbecco's modified Eagle's medium (DMEM) at $5 \mathrm{mg} / \mathrm{mL}$ and filtered through $0.22 \mu \mathrm{m}$ filters before $1 \times 10^{7}$ viable cells $/ \mathrm{mL}$ was suspended in culture medium. Following this, $100 \mu \mathrm{L}$ of the solution was dispensed per well in 96-well flat-bottomed tissue-culture plates. Cells were allowed to grow with and without PrxII and control siRNA to determine the difference between cell proliferations. The $96-$ well plates were incubated at $37^{\circ} \mathrm{C}$ in an incubator for 48 hours. After this, the medium in each well was removed and $100 \mu \mathrm{L}$ fresh medium added. MTT solution $(10 \mu \mathrm{L})$ was then added to each well and the plates incubated for another 4 hours at $37^{\circ} \mathrm{C}$. During this period, formazan crystals formed at the bottom of the wells. The solution in each well was aspirated and $100 \mu \mathrm{L}$ dimethyl sulfoxide (DMSO) was added to each well and mixed thoroughly to dissolve the crystals. Absorbance was read at $570 \mathrm{~nm}$. 
The experiments were repeated at least three times. Cell proliferation was calculated as proliferation percentage:

$$
\text { Proliferation } \%=\frac{\text { ABS with siRNA }}{\text { ABS without siRNA }} \times 100 \% \text {, }
$$

in which "ABS" represents absorbance.

\section{Calcium assay}

Determination of calcium content was accomplished by fluorometric determination. Cell pellets were suspended in a $\mathrm{Ca}^{2+}$ and $\mathrm{Mg}^{2+}$-free Hanks' solution. Intracellular $\mathrm{Ca}^{2+}$ concentration was determined by fluorescent assay using a PerkinElmer fMax microplate reader (Molecular Devices, Sunnyvale, CA, USA) and a Twinkle LB 970 Microplate Fluorometer reader (Berthold Technologies $\mathrm{GmbH} \& \mathrm{Co}$. Bad Wildbad, Germany). The method is based on the formation of a fluorescent chelate between $\mathrm{Ca}^{2+}$ and calcein fluorophore. The calcein solution was made by dissolving $0.01 \mathrm{mM}$ calcein in a $0.8 \mathrm{M}$ potassium hydroxide solution. The experiments were repeated at least three times.

\section{PSH and GSH assay}

The cell pellets were sonicated in $1 \mathrm{~mL}$ of cold buffer $(50 \mathrm{mM}$ phosphate, $\mathrm{pH}$ 6-7, containing $1 \mathrm{mM}$ ethylenediaminetetraacetic acid [EDTA]). They were then centrifuged at 10,000 g for 15 minutes at $4^{\circ} \mathrm{C}$. The supernatant was removed, stored on ice, and treated with $7 \%$ sulfosalicylic acid containing $0.1 \mathrm{mM}$ EDTA. The mixture was centrifuged at maximum speed for 10 minutes at $4^{\circ} \mathrm{C}$. Afterwards, the supernatant was collected for $\mathrm{GSH}$ assay. The precipitated pellets were washed three times with $7 \%$ sulfosalicylic acid containing $0.1 \mathrm{mM}$ EDTA buffer. The denatured protein was dissolved in $0.1 \mathrm{M} \mathrm{pH} 7.5$ phosphate buffer containing $0.1 \mathrm{mM}$ EDTA to assay for PSH concentration. Total levels of PSH and GSH were measured using a Glutathione Assay Kit from Cayman Chemical (Ann Arbor, MI, USA), which utilizes a carefully optimized enzymatic recycling method to quantify the sulfhydryl group level. The sulfhydryl group of PSH and GSH reacts with 5,5'-dithiobis-2-nitrobenzoic acid (DTNB; Ellman's reagent) and produces a yellow-pigmented 5-thio-2-nitrobenzoic acid (TNB). Measurement of the absorbance of TNB at $405 \mathrm{~nm}$ provides an accurate estimation of PSH and GSH in the sample. The experiments were repeated at least three times. The experiment was performed in quadruple replicates each time.

\section{Phosphate assay}

Quantitation of inorganic phosphate (Pi) in solution was performed using a Phosphate Fluorometric Assay Kit from
BioVision, Inc. (Milpitas, CA, USA). In this assay, Pi reacted with maltose to produce glucose in the presence of a special enzyme. The glucose is specifically oxidized to generate a product that reacts with the OxiRed ${ }^{\mathrm{TM}}$ fluorophore to generate fluorescence with the excitation wavelength at $535 \mathrm{~nm}$ and the emission wavelength at $587 \mathrm{~nm}$. The fluorescent assay was performed using the Twinkle LB 970 Microplate Fluorometer reader. The experiments were repeated at least two times.

\section{Results}

\section{Prx PSH status in radioresistant breast-cancer cells}

PrxII belongs to a family of detoxification enzymes that use cysteine as an active site to reduce peroxides. PrxI, -II, and -III isozymes are typical 2-Cysteine (2Cys) Prxs and contain two conserved cysteine residues, the peroxidatic cysteine (generally near residue 50), and the resolving cysteine (near residue 170). ${ }^{19}$ During the catalysis, the peroxidatic cysteine, a redox-sensitive reactive site, becomes oxidized, forming disulfide in the presence of ROS generated by an oxidative environment. GSH has been known to provide the cells with a reducing environment in addition to maintaining the proteins in a reduced state. ${ }^{20}$ The oxidized PrxII is then reduced in the reducing environment to regenerate the active PrxII. This may be an important redox regulatory mechanism through the alteration of cellular thiol. PrxII has been shown to be overexpressed in radioresistant breast-cancer cells. The thiol status of PrxII might be related to the cellular oxidative environment and cellular resistance level to the environmental oxidants. Here, we performed immunoblot analyses to analyze the Prx thiol status in MCF+FIR3 radioresistant breast-cancer cells and MCF+FIS4 radiosensitive cells using PrxI, -II, and -III antibodies in the presence and absence of $0.1 \mathrm{mM}$ DTT reducing agent. The results are shown in Figure 1.

An increased amount of PrxI and PrxII dimers was observed in radioresistant $\mathrm{MCF}+\mathrm{FIR} 3$ breast-cancer cells when compared with MCF+FIS4 radiosensitive cells (Figure 1A and B). The dimers were formed through disulfide bonds between the peroxidatic cysteine in the first subunit and the resolving cysteine located at the $\mathrm{C}$-terminus of another subunit in the nonreducing environment. ${ }^{21}$ Unlike PrxI and -II, the level of PrxIII dimer was seen to be higher in the MCF+FIS4 radiosensitive cells (Figure 1C). It is likely that the location differentiates the response of PrxI, -II, and -III to IR. Both PrxI and PrxII are cytosolic proteins. PrxI is also found in the nucleus and PrxII is also associated with the membrane. PrxIII is exclusively located in the mitochondria. The higher dimerization level of PrxIII in MCF+FIS3 sensitive cells 


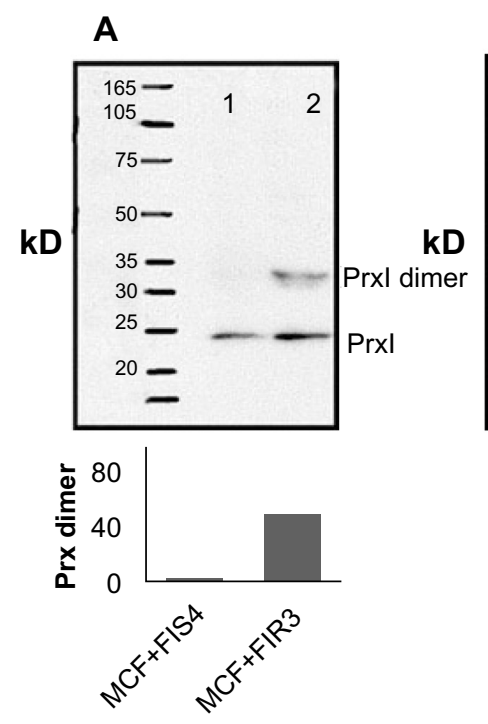

\section{B}
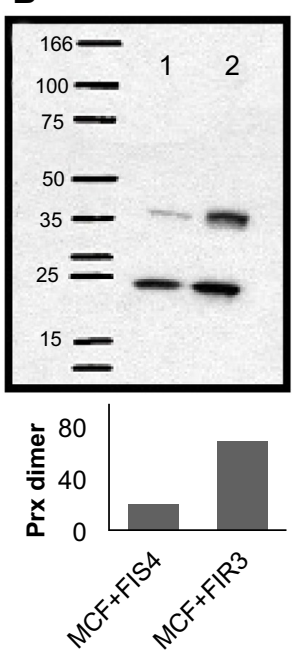

E
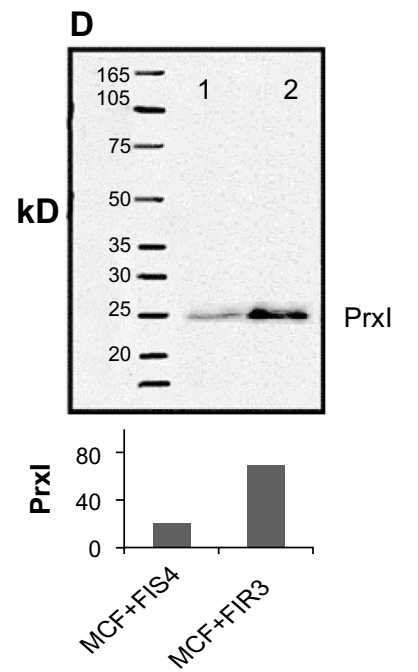

G

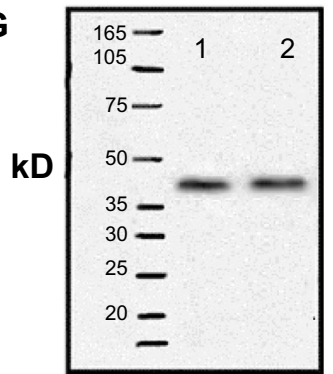

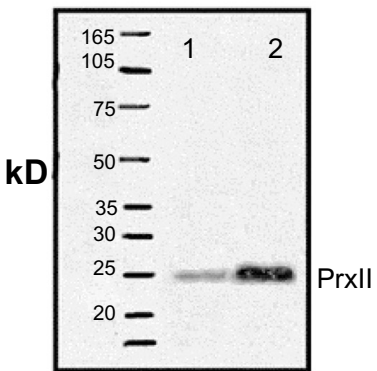

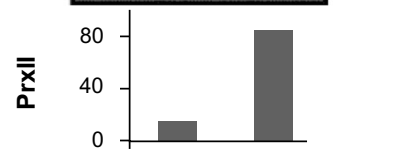

$\beta$-actin

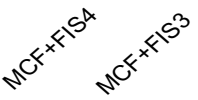

H

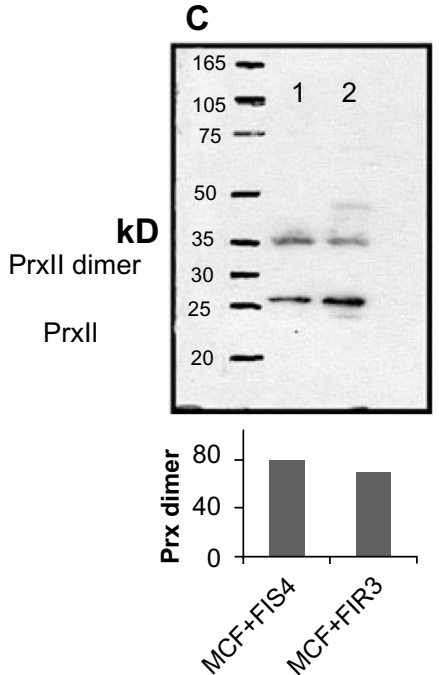

PrxIII dimer

PrxllI
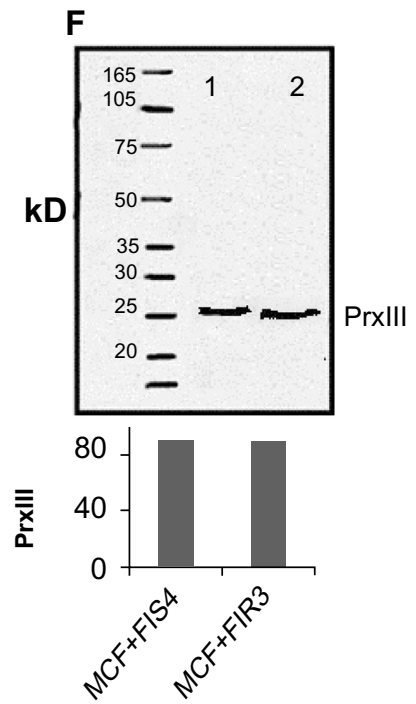

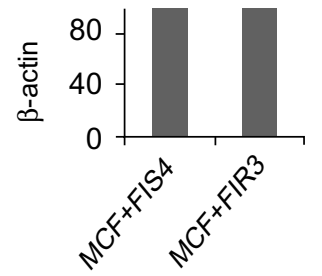

Figure I Immunoblot analysis of peroxiredoxin (Prx) I, II, and III in MCF+FIS3 radiosensitive breast-cancer cells and MCF+FIR3 radioresistant breast-cancer cells in the presence and absence of dithiothreitol (DTT) reducing agent. Lane I is the result for MCF+FIS4 radiosensitive cells. Lane 2 is the result for MCF+FIR3 radioresistant cells. (A-C) Immunoblot results for Prxl, -II, and -III in the absence of DTT reducing agent; (D-G) immunoblot results for Prxl, -II, and -III in the presence of DTT reducing agent; (H) control experiment for the immunoblot analyses using the $\beta$-actin antibody.

could possibly result from baseline exposure to the oxidative environment found in the mitochondria, even in the absence of radiation. The increased levels of PrxI and -II dimers in $\mathrm{MCF}+\mathrm{FIR} 3$ radioresistant cells were mainly due to the prolonged exposure of MCF-7 cells to IR.
The immunoblot analyses in the reducing condition were performed alongside the just-mentioned experiments. Figure 1D-F display the immunoblot results of PrxI, -II, and -III in MCF+FIS4 radiosensitive and MCF+FIR3 radioresistant cells. The inter-subunit disulfide bonds were reduced and 
PrxI, -II, and -III monomers generated. The control experiment for the immunoblot is shown in Figure 1G. The formation of intra- and inter-molecular disulfides between protein cysteines is believed to be associated with the stabilization of extracellular proteins, protection of proteins against irreversible oxidation of critical cysteine residues, and regulation of enzyme functions and transcription. ${ }^{22}$ The results from this experiment suggest that the formation of Prx dimers in $\mathrm{MCF}+\mathrm{FIR} 3$ radioresistant breast-cancer cells was an adaptive response of the MCF-7 breast-cancer cells to the oxidative environment created by chronic irradiation.

\section{Cellular toxicity of MCF+FIR3 radioresistant cells after inhibition of Prxll expression}

In the present study, the toxicological implication of PrxII-siRNA gene inhibition in MCF+FIR3 resistant and $\mathrm{MCF}+\mathrm{FIS} 4$ sensitive cells was investigated. We were interested in studying how radioresistant and radiosensitive breast-cancer cells respond to IR when PrxII expression is knocked down. The siRNA was used to modulate the PrxII expression levels in the MCF+FIR3 and MCF+FIS4 cells in this experiment. Cytotoxicity assays of $\mathrm{MCF}+\mathrm{FIR} 3$ radioresistant cells and MCF+FIS4 radiosensitive cells treated by control or PrxIIsiRNA in the presence and absence of IR were examined. The results are shown in Figure 2. We found that cell proliferation was not significantly affected by the treatment of PrxII-siRNA in MCF+FIS4 radiosensitive cells (Figure 2A and B). This indicated that alteration of PrxII levels in MCF+FIS4 cells did not have any significant impact on cell proliferation or cell death. Figure 2C and D display the results of cell proliferation for
MCF+FIS4 radiosensitive cells treated with siRNA combined with IR. Cell proliferation in MCF+FIS4 cells pretreated with PrxII-siRNA or control siRNA was significantly reduced after IR. The reduced cell proliferation was mainly due to the radiation sensitivity of MCF+FIS4 cells (data not shown). PrxII-siRNA did not appear to play a significant role in the decrease of cell proliferation after IR, although the resistance relative to the sensitive cells was correlated.

Figure 2E displays the cell proliferation results in $\mathrm{MCF}+\mathrm{FIR} 3$ radioresistant breast-cancer cells treated with control and PrxII-siRNA. PrxII expression levels were monitored by immunoblot analyses (Figure 2F). There was no significant change in cell proliferation for PrxII-siRNA treatment versus control siRNA treatment in $\mathrm{MCF}+\mathrm{FIR} 3$ resistant cells. However, combined treatment with PrxII-siRNA and IR did enhance the cellular toxicity induced by radiation in comparison to the control experiments in MCF+FIR3 cells, as shown in Figure 2G. The immunoblot analyses ensured the PrxII expression was suppressed by PrxII-siRNA (Figure 2H). The results indicated that inhibition of PrxII gene expression by siRNA greatly reduced cell viability in response to IR. The combination of PrxII-siRNA and radiation treatment, which increased cellular toxicity induced by radiation, resulted in the increased radiosensitivity of MCF+FIR3 breast-cancer cells.

\section{Cellular toxicity and cellular thiol status in the radioresistant breast-cancer cells after silencing Prxll gene expression}

GSH is a major player in maintaining the cellular reducing environment. A PrxII dimer with a disulfide linkage can be reduced in the reducing environment with the

A

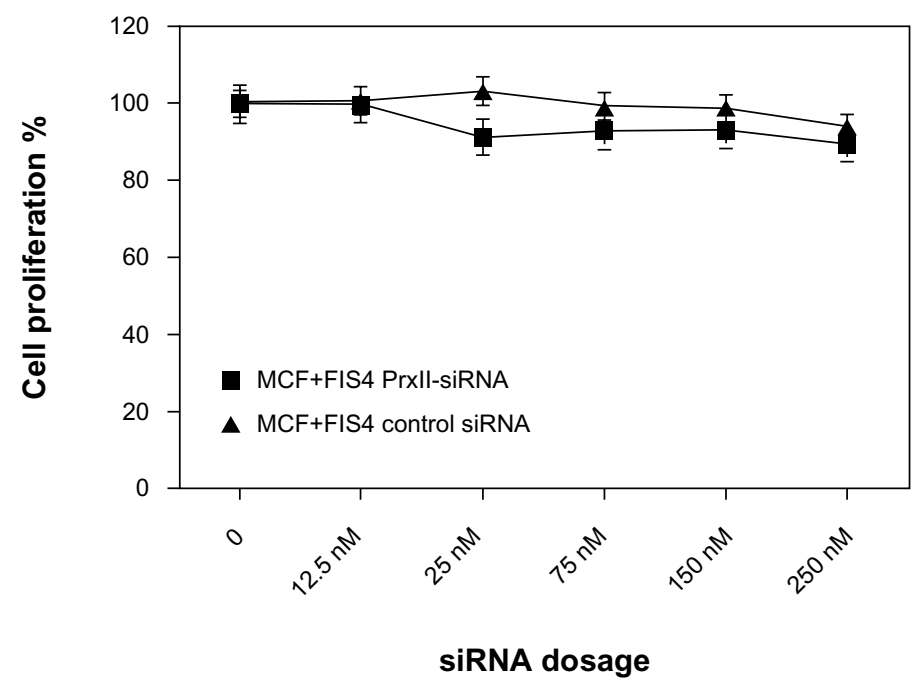

Figure 2 (Continued) 
B Immunoassay for A experiment.

Immunoblot analysis of control siRNA treated cells
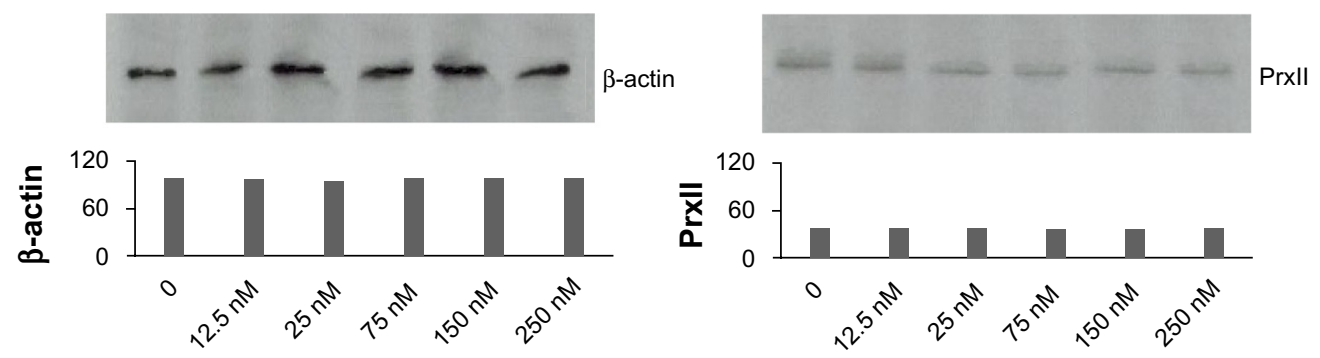

Immunoblot analysis of Prxll-siRNA treated cells

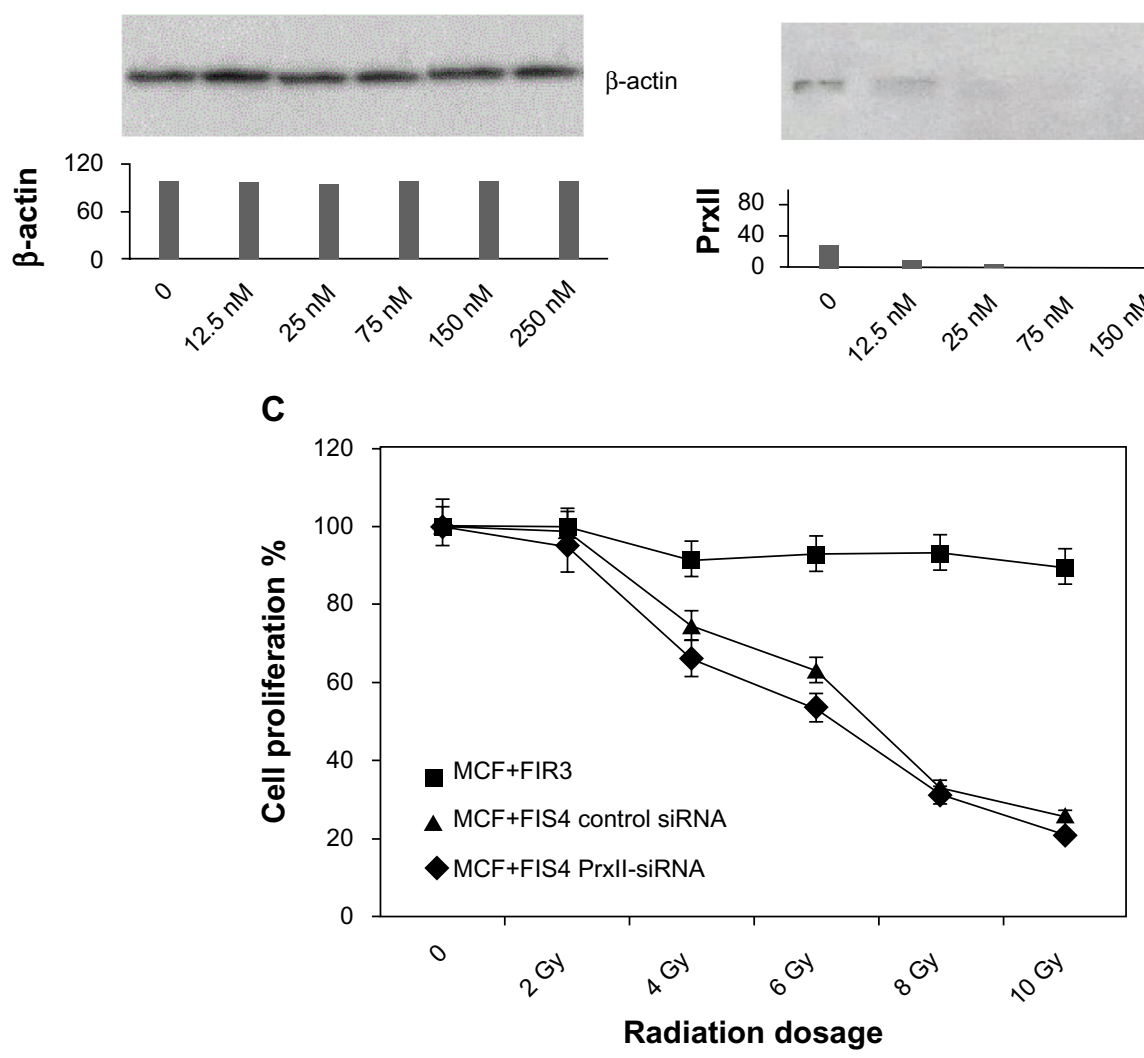

D Immunoblot analysis for $\mathrm{C}$ experiment
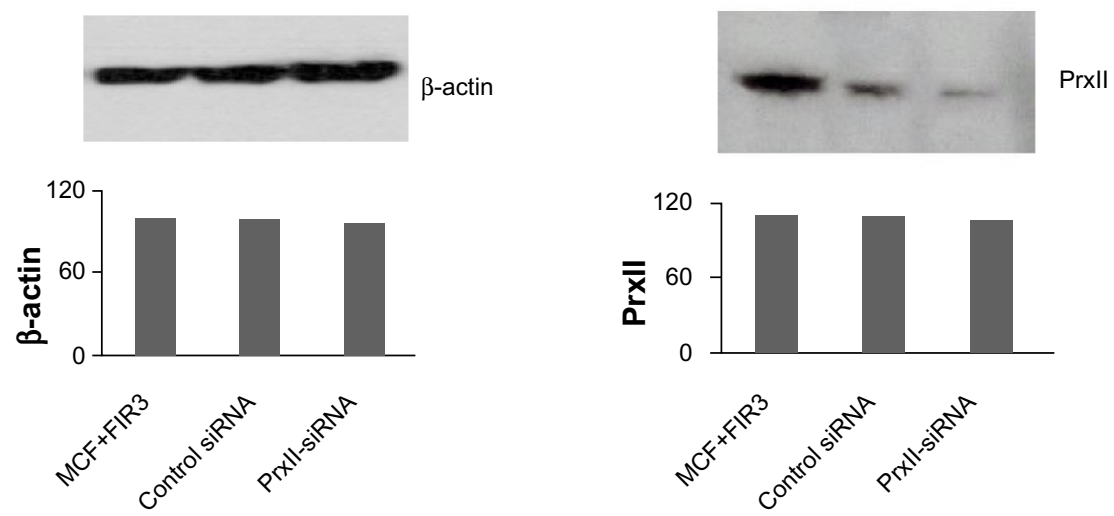

Figure 2 (Continued) 


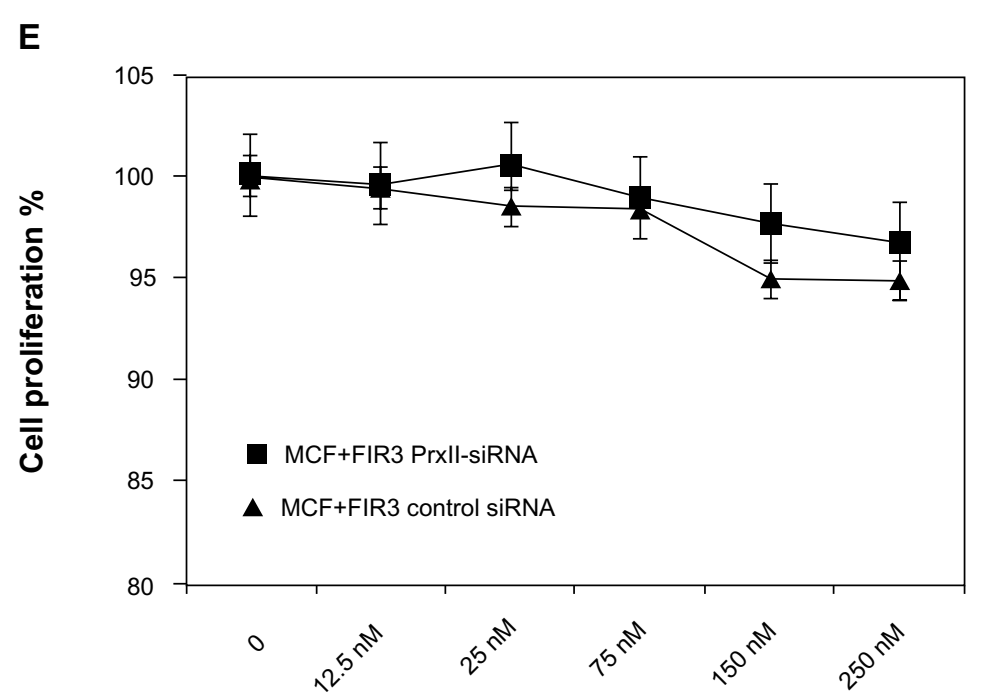

siRNA dosage

F Immunoassay for E experiment

Immunoblot analysis of control siRNA treated cells

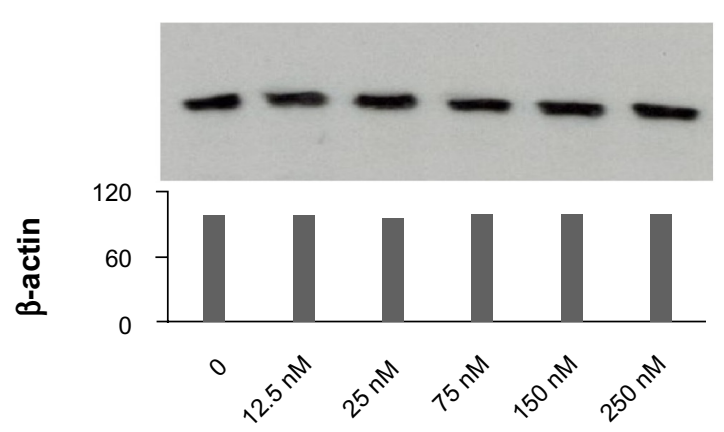

$\beta$-actin

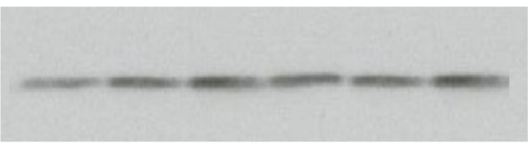

Prxll

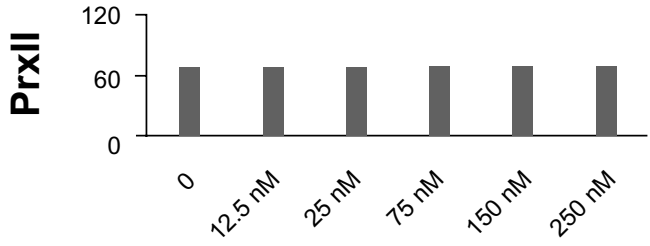

Immunoblot analysis of Prxll-siRNA treated cells
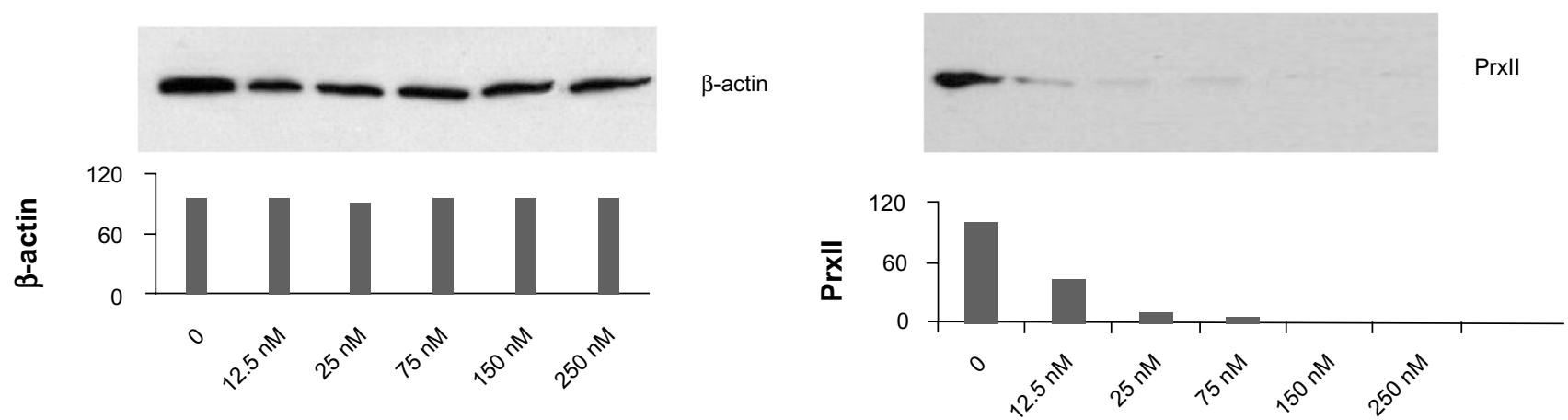

Figure 2 (Continued) 
G

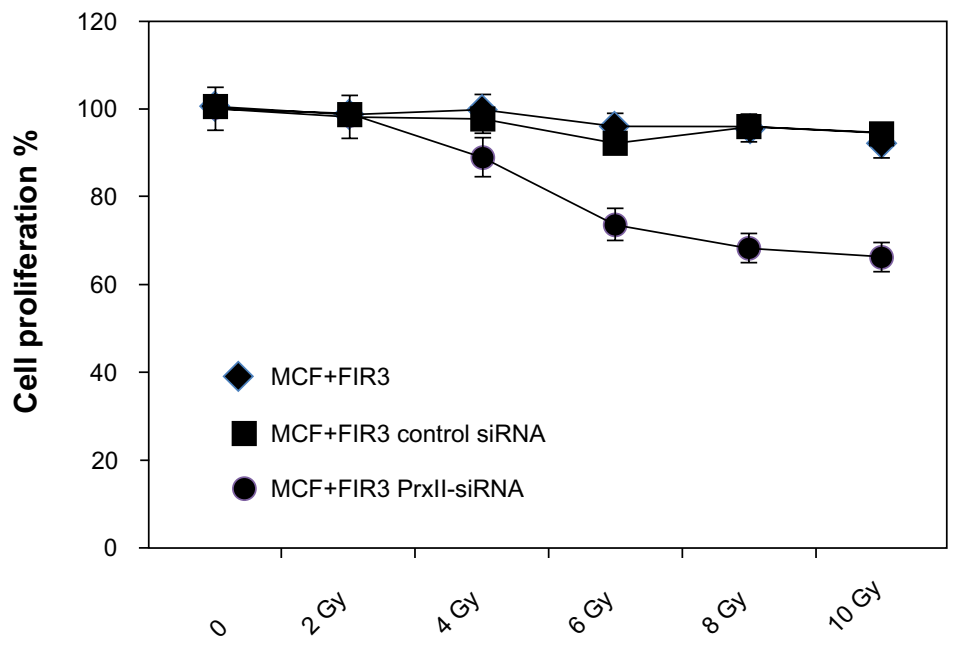

Radiation dosage

\section{H Immunoblot analysis for G experiment}
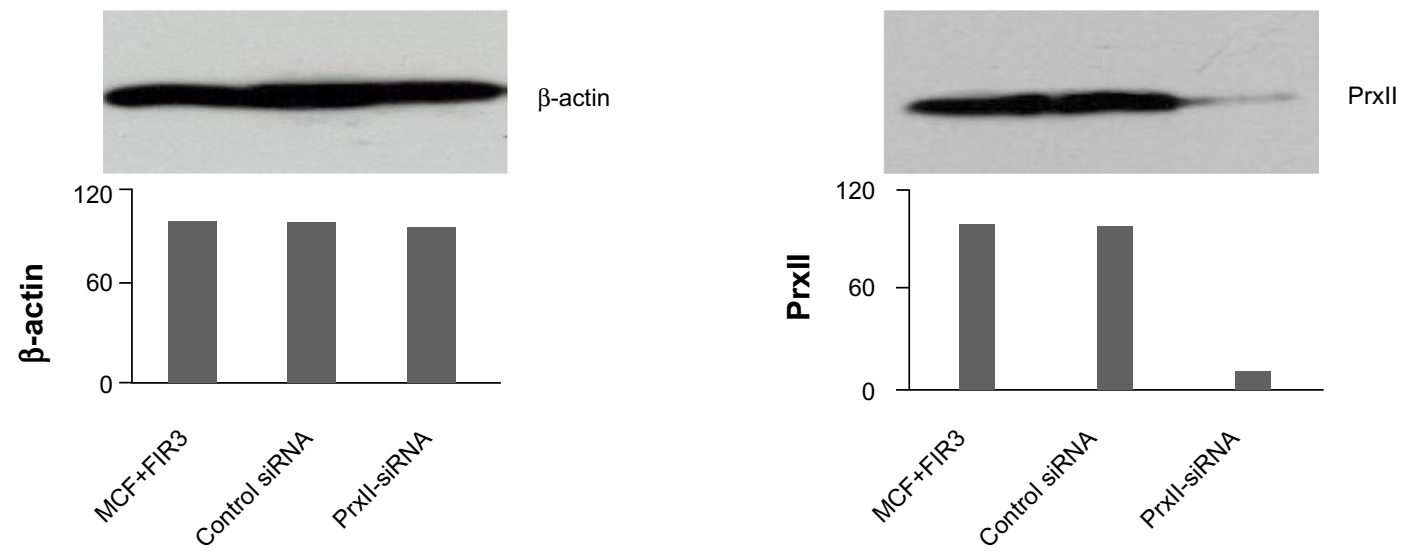

Figure 2 Cell proliferation of MCF+FIS4 radiosensitive and MCF+FIR3 radioresistant breast-cancer cells in response to radiation and small interference RNA (siRNA) treatment. (A) Cell proliferation of MCF+FIS4 radiosensitive cells following the transfection of siRNA at concentrations 0.0, I2.5, 25.0, 75.0, I50.0, and 250.0 nM. ( $\Delta$ ) Result for the cells treated with control siRNA. ( $\boldsymbol{\square})$ Result for the cells treated with peroxiredoxin (Prx) II-siRNA. (B) Immunoblot results for $\beta$-actin and Prxll from cells transfected with control siRNA and cells transfected with Prxll-siRNA. (C) Cell proliferation results for MCF+FIS4 radiosensitive cells with combined treatment of 25 nM siRNA for 72 hours and ionizing radiation (IR). ( $\bullet$ Result for the cells treated with combined Prxll-siRNA and IR. ( $(\boldsymbol{\Delta})$ Result for cells treated with combined control siRNA and IR. ( $\mathbf{\square})$ Result for the MCF+FIR3-resistant cells used for comparison analysis. (D) Immunoblot analysis undertaken to monitor Prxll expression levels after control siRNA and Prxll-siRNA transfection. (E) Cell proliferation for MCF+FIR3 radiosensitive cells following the transfection of siRNA at concentrations 0.0 , I2.5, 25.0, 75.0, I50.0, and $250.0 \mathrm{nM}$. ( $\mathbf{\Delta})$ Result for the cells treated with control siRNA. ( $\boldsymbol{\bullet})$ Result for the cells treated with Prxll-siRNA. (F) Prxll expression levels were monitored by immunoblot analysis. (G) Cell proliferation results for MCF+FIR3 radioresistant cells treated with combined siRNA ( $25 \mathrm{nM})$ for 72 hours and IR. (•) Result for the cells treated with combined Prxll-siRNA and IR. ( $\bullet$ ) Result for cells treated with control siRNA and IR. ( $\bullet$ ) Cell proliferation results for untreated MCF+FIR3 radioresistant cells. (H) Prxll expression levels were monitored by immunoblot analyses after control siRNA and Prxll-siRNA transfection.

presence of GSH. GSH and PSH have been shown to be associated with many redox-inducing reagents and chemotherapeutic drugs. We were interested in discovering whether the PrxII-siRNA that inhibits PrxII gene expression would affect the GSH and PSH levels and to see if these changes were directly reflected in intracellular thiol alterations. Since pretreatment with PrxII-siRNA did not show significant effects on cell proliferation in MCF+FIS4 radiosensitive cells in response to IR (Figure 2), the focus of this study was the radiation response of MCF+FIR3 radioresistant cells under the condition of inhibited PrxII expression. We hypothesized that there may be a relationship between PrxII level and cellular PSH and GSH levels in MCF+FIR3 radioresistant cells. 
siRNA technology has become a powerful research tool to target molecules that conventional therapeutics cannot and is being actively pursued for its therapeutic potential. ${ }^{23-25}$ In this experiment, PrxII-siRNA was used to silence PrxII gene expression in comparison to the control siRNA that did not interfere with PrxII expression. A TNB assay was used to measure the cellular PSH and GSH levels. The disulfide was reduced by GSH reductase to recycle the PSH and GSH and produce more TNB. The rate of TNB production was directly proportional to this recycling reaction, which in turn was directly proportional to the concentration of PSH and GSH in the sample. Figure 3 shows the relative GSH and PSH levels in MCF+FIR3 radioresistant and MCF+FIS4 radiosensitive breast-cancer cells. The data were standardized by the total protein concentration of the cell extract. Relative GSH levels in MCF+FIR3 resistant and MCF+FIS4 sensitive cells are shown in Figure 3A, while PSH levels are shown in Figure 3B. Both GSH and PSH levels are higher in MCF+FIR3 resistant cells than in MCF+FIS4 radiosensitive cells. This result correlates well with the increased PrxII level in the MCF+FIR3 radioresistant cells in comparison to the MCF+FIS4 sensitive cells. PrxII expression levels in radioresistant and radiosensitive cells are shown in Figure 3C and D. The lower GSH, PSH, and PrxII levels in MCF+FIS4 cells could be one of the factors that renders the cells more sensitive to radiation.

Figure 4A and B display the results from the experiments in which the GSH and PSH levels in MCF+FIR3 radioresistant cells were measured after the treatment of control siRNA in comparison to combined control siRNA and IR treatment. The results showed that when PrxII was overexpressed in MCF+FIR3 resistant cells, the GSH and PSH levels were not affected significantly by IR treatment (Figure 4C). GSH and PSH levels in MCF+FIR3 radioresistant cells pretreated with PrxII-siRNA that inhibited PrxII gene expression are shown in Figure 4D and E. In contrast to the result of the control siRNA experiment, GSH and PSH levels were significantly reduced by IR when PrxII gene expression was knocked down (Figure 4F). It appears that there is a connection between cellular radioresistance levels and cellular thiol status. The results indicate that the increased cellular toxicity - induced by IR in MCF+FIR3 radioresistant cells pretreated with PrxII-siRNA - that inhibited PrxII expression occurs through lowering cellular GSH and PSH levels.
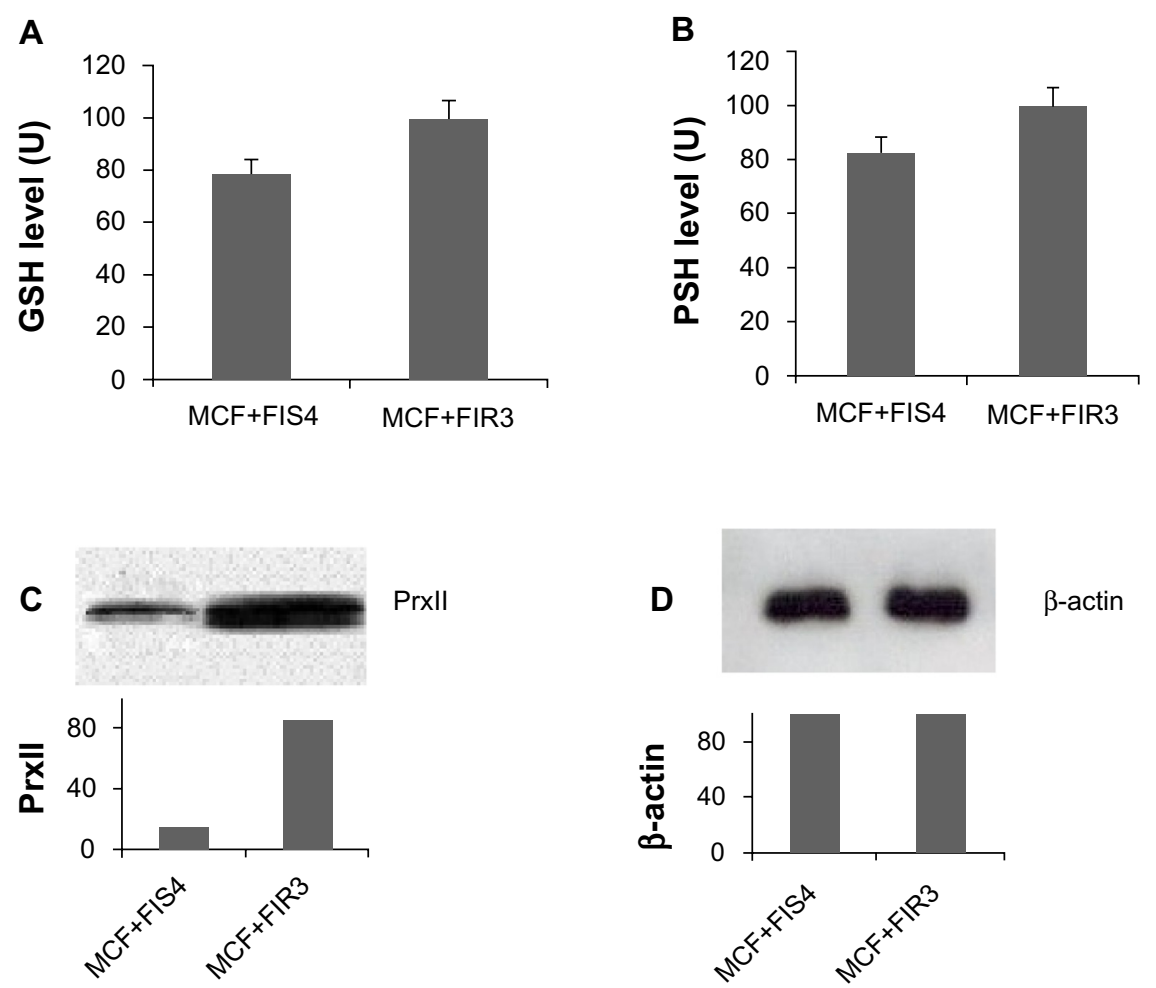

Figure 3 Glutathione (GSH) and protein thiol (PSH) levels in radioresistant breast-cancer cells and radiosensitive breast-cancer cells. (A) GSH levels in MCF+FIS4 sensitive cells and MCF+FIR3 resistant cells. (B) PSH levels in MCF+FIS4 sensitive cells and MCF+FIR3 resistant cells. (C) Immunoblot analysis of peroxiredoxin (Prx) II in MCF+FIS4 and MCF+FIR3 cells. (D) Immunoblot analysis of $\beta$-actin in MCF+FIS4 and MCF+FIR3 cells. 
A

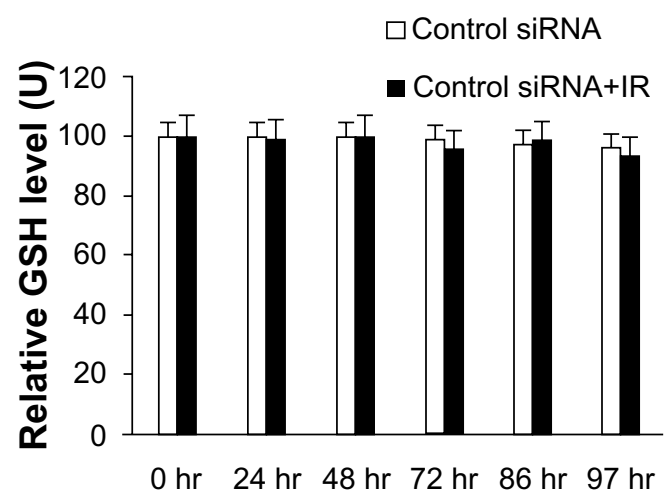

Hours after siRNA transfection

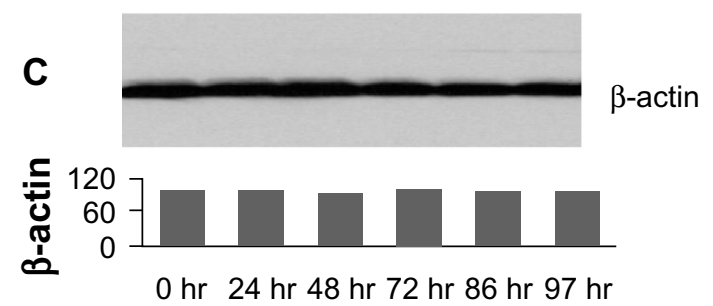

D

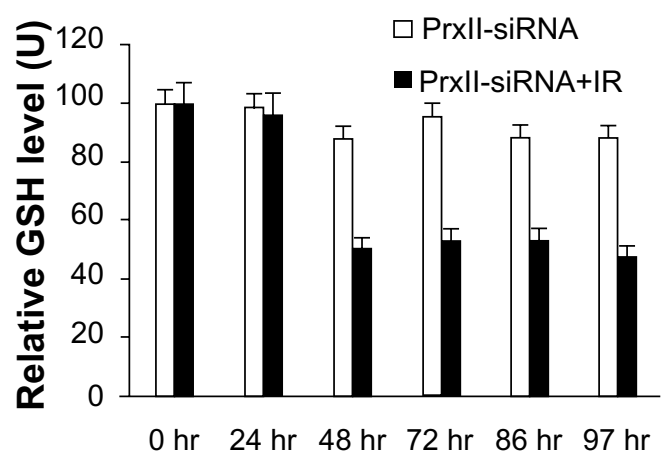

Hours after siRNA transfection
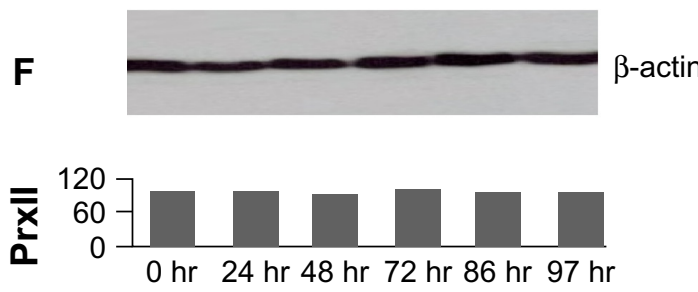

B

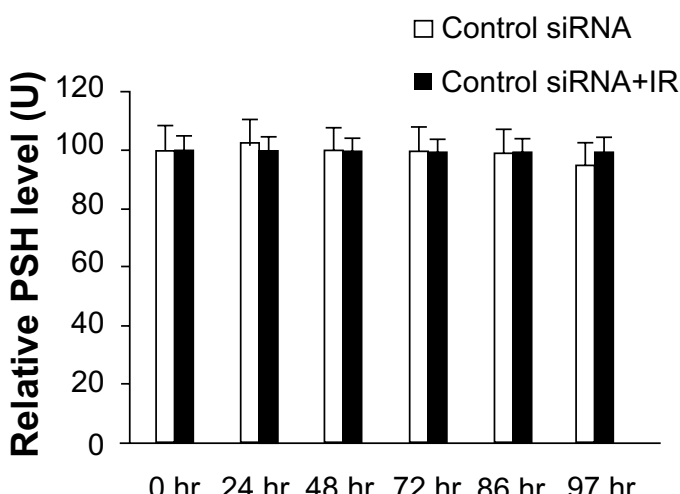

Hours after siRNA transfection

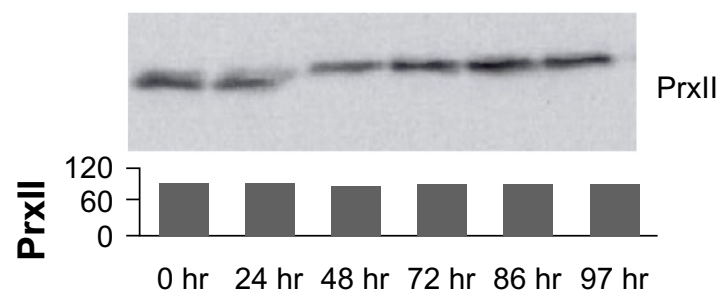

E

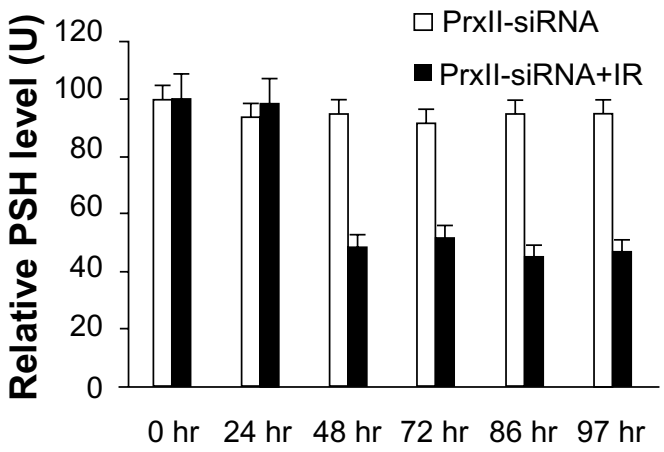

Hours after siRNA transfection

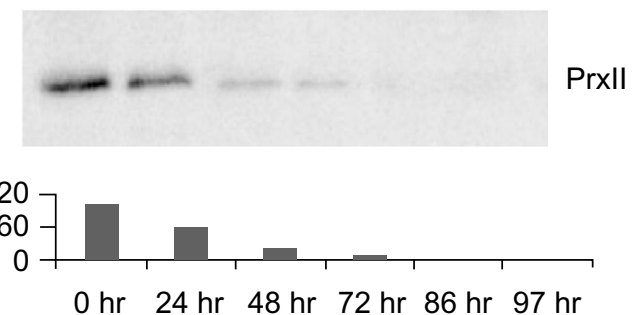

Figure 4 Glutathione (GSH) and protein thiol (PSH) levels in MCF+FIR3 radioresistant cells pretreated with small interference RNA (siRNA), with and without subsequent 5 Gy ionizing radiation (IR). (A) GSH levels with control siRNA pretreatment, with and without subsequent 5 Gy IR. ( $\square$ ) Result for cells treated with control siRNA. (घ) Result for cells treated with combined control siRNA and IR. (B) PSH levels pretreated with control siRNA, with and without subsequent 5 Gy IR. ( $\square$ ) Result for the cells treated with peroxiredoxin (Prx) II-siRNA. (口) Result for cells treated with combined Prxll-siRNA and IR. (C) Results of immunoblot analysis undertaken to monitor inhibition of Prxll expression by Prxll-siRNA. (D) GSH levels with Prxll-siRNA pretreatment, with and without subsequent 5 Gy IR. ( $\square$ ) Result for cells treated with Prxll-siRNA. (口) Result for cells treated with combined Prxll-siRNA and IR. (E) PSH levels with Prxll-siRNA pretreatment, with and without subsequent 5 Gy IR. ( $\square$ ) Result for cells treated with Prxll-siRNA. (घ) Result for cells treated with combined Prxll-siRNA and IR. (F) Results of immunoblot analysis undertaken to monitor inhibition of Prxll expression by Prxll-siRNA. Abbreviation: hr, hours. 


\section{Impairment of the plasma membrane $\mathrm{Ca}^{2+}$ pump in Prxll-siRNA-treated radioresistant breast-cancer cells as a consequence of perturbation of the cellular thiol homeostasis by IR}

We were interested in investigating whether the loss of cellular thiol induced by IR as a result of PrxII-siRNA gene inhibition impairs the activity of the plasma membrane $\mathrm{Ca}^{2+}$ pump. The $\mathrm{Ca}^{2+}$ level was measured with sequential addition of carbonyl cyanide m-chlorophenyl hydrazone (CCCP), digitonin, and A23187 compounds. "CCCP" is a protonophore that dissipates the mitochondrial membrane potential, releases sequestered mitochondrial calcium stores, and irreversibly prevents mitochondrial calcium uptake. Digitonin makes the plasma membrane permeable without affecting the mitochondrial and endoplasmic reticular membrane, while the A23187 $\mathrm{Ca}^{2+}$ ionophore makes all cellular compartments permeable.

The rationale for this experiment was that cells were exposed to $\mathrm{CCCP}$, which released $\mathrm{Ca}^{2+}$ from mitochondria to cytosol. The subsequent addition of digitonin would release the $\mathrm{Ca}^{2+}$ from cytosol to the extracellular space, while keeping the mitochondrial and endoplasmic reticular membranes intact. Adding A23187 would release the remaining intracellular $\mathrm{Ca}^{2+}$, thus allowing the measurement of the total cellular $\mathrm{Ca}^{2+}$ released.$^{26} \mathrm{As}$ shown in Figure 5A, the total $\mathrm{Ca}^{2+}$ released from the cytosol by the sequential addition of CCCP, digitonin, and A23187 was not significantly affected in MCF+FIR3 radioresistant cells transfected with control siRNA after IR when PrxII gene expression was not inhibited (Figure 5B). This result shows that the released $\mathrm{Ca}^{2+}$ from the cytosol could be extruded into the extracellular space by membrane $\mathrm{Ca}^{2+}$ adenylpyrophosphatase (ATP) when PrxII was overexpressed in MCF+FIR3 resistant cells, suggesting that the plasma membrane pump $\mathrm{Ca}^{2+}$-ATPase was functional so could extrude the $\mathrm{Ca}^{2+}$ from cytosol to the extracellular space after IR.

When PrxII gene expression was inhibited by PrxIIsiRNA transfection, a significant increase in $\mathrm{Ca}^{2+}$ level was observed after IR in MCF+FIR3 radioresistant cells with the sequential addition of CCCP and A23187 (Figure 5C and D). The increase in $\mathrm{Ca}^{2+}$ level was also seen in cells treated with A23187, CCCP, and digitonin (Figure 5C). The amount of $\mathrm{Ca}^{2+}$ mobilized by CCCP in MCF7+FIR3 radioresistant breast-cancer cells transfected by PrxII-siRNA, not excreted from the cells following IR, was markedly increased compared with in the control cells treated with the control siRNA, indicating that the plasma membrane $\mathrm{Ca}^{2+}$-extruding mechanism was inhibited. This result suggests that IR had a significant impact on the $\mathrm{Ca}^{2+}$ membrane extrusion mechanism after the silencing PrxII gene expression by PrxII-siRNA in MCF+FIR3 resistant cells.

Since plasma membrane $\mathrm{Ca}^{2+}$-ATPase is known to play a critical role in regulating cytosolic free $\mathrm{Ca}^{2+}$ concentration, it was of interest to investigate whether this impairment of $\mathrm{Ca}^{2+}$ efflux by PrxII-siRNA following IR was associated with an enhanced cytosolic free $\mathrm{Ca}^{2+}$ level. To monitor cytosolic $\mathrm{Ca}^{2+}$ level in MCF7+FIR3 radioresistant cells transfected with PrxII-siRNA following IR, the level of Pi was monitored. The Pi level in normal blood is very similar to that of calcium. The use of this activity as an index of cytosolic $\mathrm{Ca}^{2+}$ level fluctuations has been previously validated ${ }^{27}$ The addition and removal of phosphate from proteins in all cells is a pivotal strategy in the regulation of metabolic processes. As shown in Figure 6A and B, an increase in the concentration of Pi was associated with IR treatment in MCF7+FIR3 radioresistant breast-cancer cells when PrxII expression was suppressed. The phosphate level in the radioresistant cells treated with control siRNA did not show any significant change; neither was there any significant change in phosphate level in the radioresistant cells treated with combined control siRNA and IR. Since PrxII was found to be associated with the cell membrane, the result may suggest that PrxII plays a role in protecting the cell membrane from IR-induced oxidative damage.

\section{Discussion}

Advanced and recurrent tumors are usually resistant to chemotherapy and radiation therapy, leading to a poor survival rate. It has been reported that while traditional chemotherapy can kill the majority of cancer cells, it fails to target CSCs. ${ }^{28}$ Moreover, initial treatment can increase the proportion of radio- and drug-resistant CSCs, resulting in disease recurrence. ${ }^{29}$ Radiotherapy is still one of the most commonly used chemotherapeutic methods for cancer recurrence treatment. The problem with this method is its lack of specificity in targeting cancer cells. Here, we augmented therapeutic IR with a targeting property to recognize certain genes inside cancer cells that are activated in adaptive response to radiation. PrxII has been shown to be upregulated in tissues isolated from patients who did not respond to radiation therapy and in radioresistant MCF+FIR3 breast-cancer cells this is associated with breast-cancer radiation sensitivity or resistance. ${ }^{30}$ This increased PrxII expression confers radioresistance to cancer cells due to its role in protecting cells from radiation-induced cell death. Prxs are important 


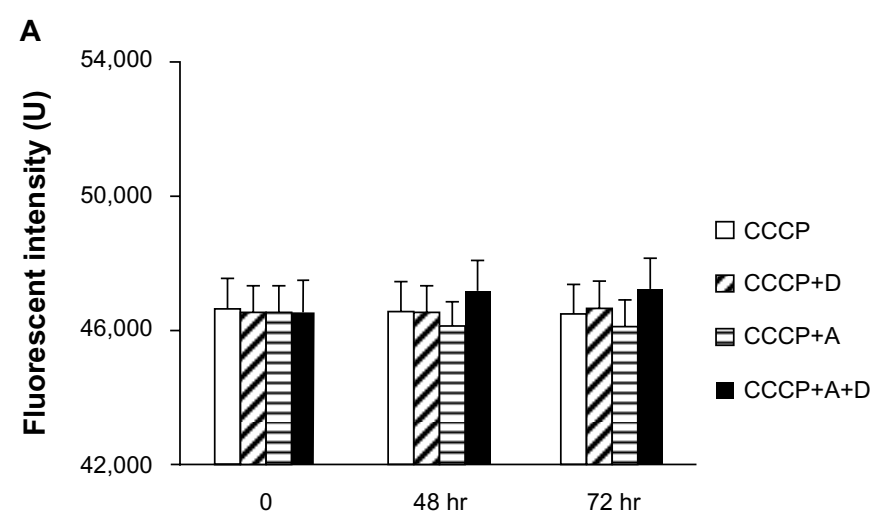

Hours after siRNA transfection

B Immunoblot analysis of control siRNA treated cells
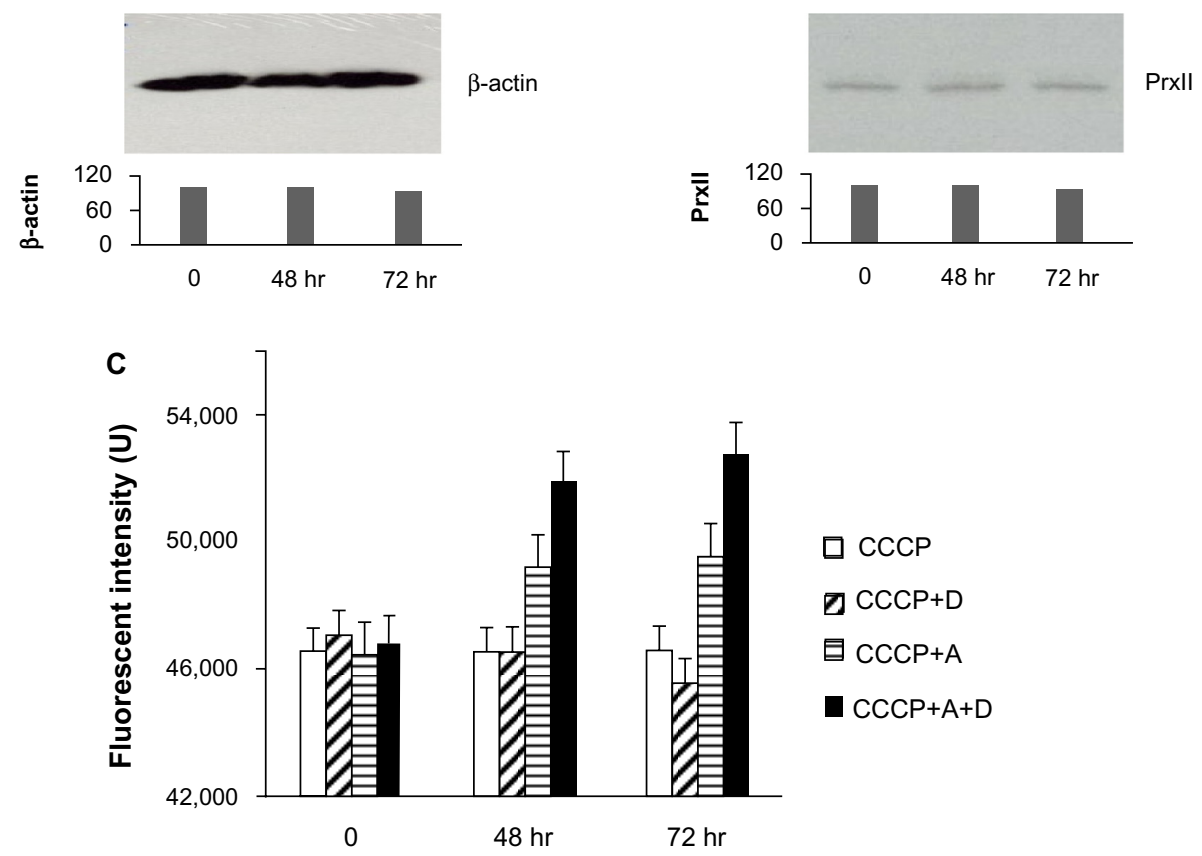

Hours after siRNA transfection

D Immunoblot analysis of Prxll-siRNA treated cells
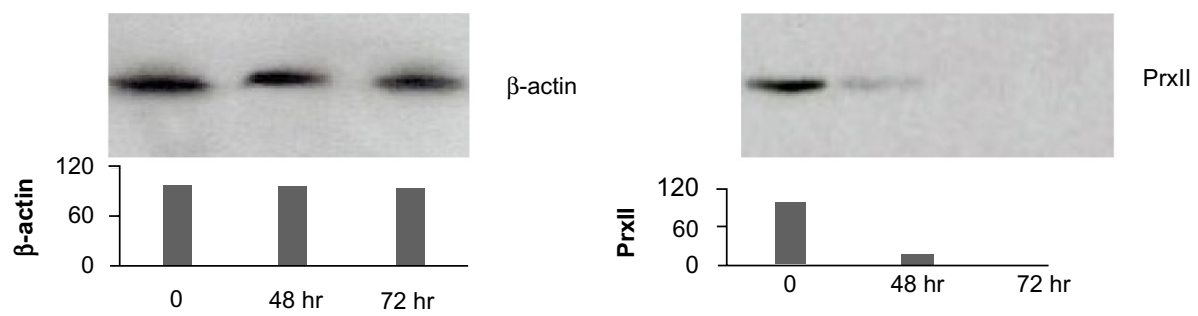

Figure 5 Effect of calcium released from MCF+FIR3 radioresistant cells transfected with control small interference RNA (siRNA) and peroxiredoxin (Prx) II-siRNA in response to ionizing radiation (IR). (A) Calcium released in response to IR for MCR+FIR3 resistant cells transfected with 25 nM control siRNA. ( $\square$ ) Cells transfected with control siRNA in response to IR with sequential addition of the carbonyl cyanide m-chlorophenyl hydrazone (CCCP), A23I87 (A), and digitonin (D). ( $\mathbf{\nabla})$ Cells transfected with control siRNA in response to IR with the sequential addition of CCCP and digitonin. ( $\boxminus$ ) Cells transfected with control siRNA in response to IR with the addition of CCCP and A23 I87. ( $\square$ ) Cells transfected with control siRNA in response to IR with the addition of CCCP. (B) Results of immunoblot analysis undertaken to monitor Prxll expression by Prxll-siRNA. (C) Calcium released in response to IR for MCR+FIR3 resistant cells transfected with 25 nM Prxll-siRNA. (口) Cells transfected with Prxll-siRNA in response to IR with sequential addition of the CCCP, A23 I87, and digitonin. ( $\mathbf{v})$ Cells transfected with Prxll-siRNA in response to IR with the sequential addition of CCCP and digitonin. $(\boxminus)$ Cells transfected with Prxll-siRNA in response to IR with the addition of CCCP and A23I87. ( $\square$ ) Cells transfected with Prxll-siRNA in response to IR with the addition of CCCP. (D) Results of immunoblot analysis undertaken to monitor inhibition of Prxll expression by Prxll-siRNA.

Abbreviation: hr, hours. 


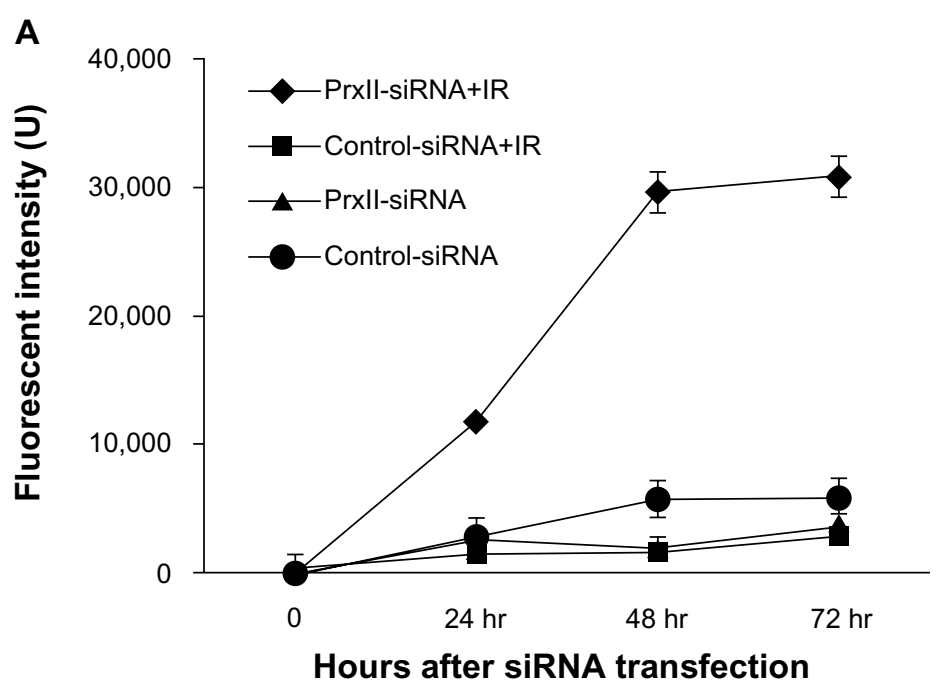

\section{B Immunoblot analysis of Prxll-siRNA treated cells}
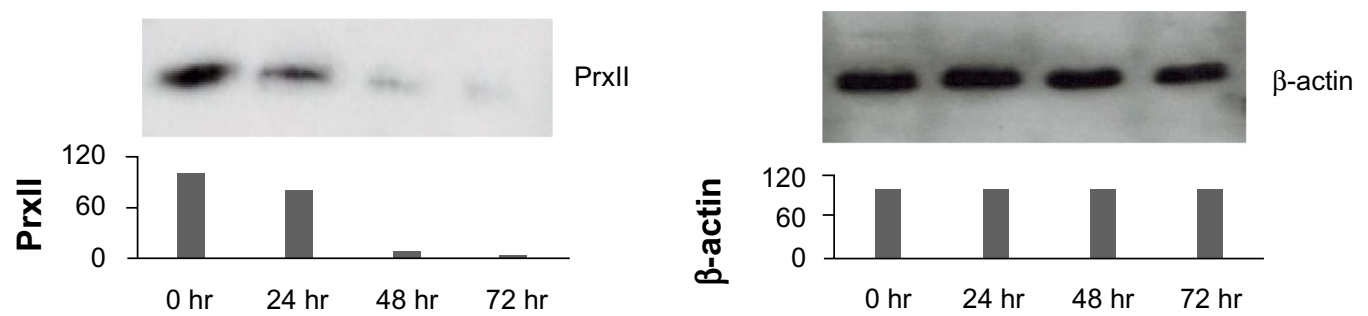

Immunoblot analysis of control siRNA treated cells
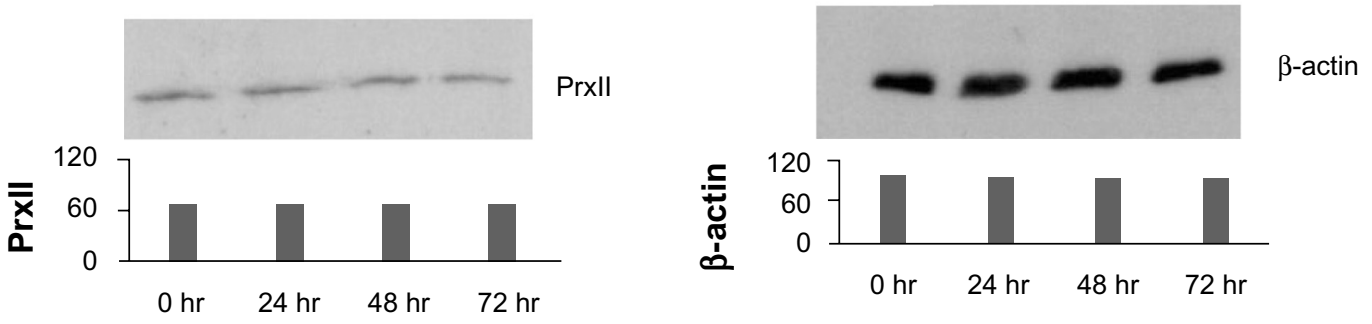

Figure 6 Intracellular phosphate levels in MCF+FIR3 radioresistant breast-cancer cells pretreated with $25 \mathrm{nM}$ of small interference RNA (siRNA) in the presence and absence of ionizing radiation (IR). (A) Fluorescent intensity of intracellular phosphate levels measured at $535 \mathrm{~nm}$ and the emission wavelength at $587 \mathrm{~nm}$. ( $\$$ ) Cells treated with

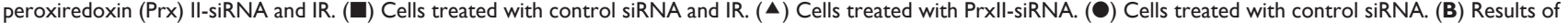
immunoblot analysis undertaken to monitor inhibition of Prxll expression by Prxll-siRNA.

Abbreviation: hr, hours.

not only as antioxidant proteins, keeping toxic $\mathrm{H}_{2} \mathrm{O}_{2}$ and peroxynitrite levels low, but also in such processes as apoptosis, differentiation, and proliferation. Therefore, alteration in PrxII level, determination of PrxII mechanisms of action, and targeting PrxII modulation by chemical or biological means may lead to new strategies that can be developed to improve radiation therapeutic ratios.

We have been using siRNA to modulate the PrxII gene expression level to determine the connection between PrxII expression level and radiosensitivity. We have shown that siRNA can be used to silence protein expression in a gene-specific manner - a process that has found widespread applications in the fields of functional genomics and drug discovery. ${ }^{31}$ It has become a powerful research tool to target molecules that conventional therapeutics cannot reach. In our studies, the siRNA that inhibited PrxII gene expression partially reversed the radioresistant phenotype of MCF-7 breast cancer. PrxII has a very important role in radioresistance of breast-cancer cells, even though it is not the sole factor responsible for the resistant phenotype. In the study presented here, we showed that inhibition of PrxII gene expression by PrxII-siRNA decreased PSH and GSH levels 
in $\mathrm{MCF}+\mathrm{FIR} 3$ radioresistant breast-cancer cells compared with the control cells. Thus, the mechanism of action of PrxII-siRNA sensitization of radioresistant breast-cancer cells involved both GSH and PSH.

It has been shown that processes involving changes in cellular thiol levels can affect $\mathrm{Ca}^{2+}$ signaling pathways that participate in a broad array of physiological responses. ${ }^{34}$ Previous studies have unequivocally established the key role of calcium as a universal second messenger. ${ }^{32,33}$ Further, it is becoming increasingly apparent that there are significant interactions between calcium and cellular thiol metabolism, and that these interactions modify a variety of proteins that participate in signaling transduction pathways and other fundamental cellular functions that determine cell life or death. Intracellular $\mathrm{Ca}^{2+}$ homeostasis is regulated by compartmentalization processes involving $\mathrm{Ca}^{2+}$ sequestration in mitochondria and the endoplasmic reticulum and $\mathrm{Ca}^{2+}$ extrusion from the cell by the plasma membrane $\mathrm{Ca}^{2+}$-ATP. When GSH and PSH levels are low, the $\mathrm{Ca}^{2+}$-ATPase pump can be inactivated due to the high level of ROS, which leads to the generation of abnormal calcium signals. This calcium imbalance may be caused by damage to the $\mathrm{Ca}^{2+}$-ATPase pump. Both nuclei and cytoplasmic $\mathrm{Ca}^{2+}$ can regulate phosphorylation and dephosphorylation of proteins and, as a result, modulate signal transduction pathways. Therefore, in the study reported here, inhibition of PrxII gene expression by PrxII-siRNA changed the cellular thiol status and disrupted the normal physiological pathways. As a result, it is very likely that it increased cellular toxicity and rendered MCF+FIR3 breast-cancer cells more sensitive to IR.

$\mathrm{Ca}^{2+}$ levels fluctuate with the change of the Pi levels. Pi has been used as an indicator for $\mathrm{Ca}^{2+}$ level. The increase in Pi levels we observed following IR in PrxII-siRNA radioresistant breast-cancer cells indicated an increased $\mathrm{Ca}^{2+}$ concentration. This is most likely due to the inhibition of the plasma membrane $\mathrm{Ca}^{2+}$ pump in the plasma membrane and the $\mathrm{Ca}^{2+}$ storage deficiency of the mitochondria and endoplasmic reticulum. In control cells, control siRNA treatment did not appear to cause any appreciable inhibition of the plasma membrane $\mathrm{Ca}^{2+}$ pump. The $\mathrm{Ca}^{2+}$ released from the mitochondria and/or endoplasmic reticulum would still be expected to produce at least a transient increase in cytosolic $\mathrm{Ca}^{2+}$ concentration and phosphate level, but it would not last long since the $\mathrm{Ca}^{2+}$ would eventually be extruded through the membrane $\mathrm{Ca}^{2+}$ pump. However, when the plasma membrane $\mathrm{Ca}^{2+}$ pump is damaged, the extrusion of $\mathrm{Ca}^{2+}$ released from the intracellular stores is impaired (PrxII-siRNA + radiation) and a significant increase of phosphate concentration then occurs. The results indicate that PrxII and cellular thiol played a protective role for the plasma membrane against IR-induced damage. The depletion of cellular thiol by PrxII-siRNA correlated well with the radiation response of $\mathrm{MCF}+\mathrm{FIR} 3$ radioresistant and MCF+FIS4 radiosensitive cells. The results suggest that perturbation of cellular thiol homeostasis by inhibition of PrxII represents a crucial step in modulating cell proliferation and differentiation. Better understanding of the mechanism may also generate novel strategies to overcome the development of MCF-7 breast-cancer resistance and further improve breast-cancer treatment.

\section{Future directions}

The results of this study may provide important clinical information on prediction of the behavior and treatment response of MCF-7 radioresistant breast-cancer cells. However, PrxIIsiRNA does not completely inhibit PrxII gene expression. The radioresistance phenotype of $\mathrm{MCF}+\mathrm{FIR} 3$ breast-cancer cells was not fully reversed by targeting the PrxII gene. Some of the more potent subpopulations of radioresistant CSCs may evade therapy and propagate, seeding new tumor growth. This problem poses a great challenge and opportunity for future cancer research. Further investigation will be necessary to elucidate in detail the molecular signaling mechanisms involved in radiation resistance in $\mathrm{BCSC}$ in order to identify alternative targets that can enhance the efficacy of radiotherapy due to its targeted property to specific cancer-related genes. However, PrxII may be potentially important in MCF-7 breast-cancer cells with an increased radioresistant phenotype.

We hope the results presented here will contribute to better understanding of the biochemical and clinical pharmacology of the combination approach of siRNA and therapeutic IR so that this technology can fulfill a strategy to target enzymes in CSCs, the DNA repair pathway, and cellular protection machinery to enhance chemosensitivity.

\section{Acknowledgment}

We would like to thank Arnold and Mable Beckman Foundation, Research, Scholarship, Creative Activities Award Program (RSCAAP), California State University Program for Education and Research in Biotechnology (CSUPERB), and National Science Foundation (NSF) \#HRD-0802628 for supporting this project. We want to thank Dr Robin Roth and Ms Angela Avatia for proofreading of the paper.

\section{Disclosure}

The authors declare no conflicts of interest in this work. 


\section{References}

1. Menaa C, Li JJ. The role of radiotherapy-resistant stem cells in breast cancer recurrence. Breast Cancer Manage. 2013;2(2):89-92.

2. American Cancer Society. Breast Cancer Facts and Figures 2007-2008. Atlanta, GA: American Cancer Society. Available from: http://www. cancer.org/Research/CancerFactsFigures/BreastCancerFactsFigures/ bcff-final-pdf. Accessed August 12, 2013.

3. Bonham VL, Warshauer-Baker E, Collins FS. Race and ethnicity in the genome era: the complexity of the constructs. Am Psychol. 2005;60(1): 9-15.

4. Phillips TM, McBride WH, Pajonk F. The response of CD24-/low/ $\mathrm{CD} 44^{+}$breast cancer-initiating cells to radiation. J Natl Cancer Inst. 2006;98(24):1777-1785.

5. Diehn M, Cho RW, Lobo NA, et al. Association of reactive oxygen species levels and radioresistance in cancer stem cells. Nature. 2009;458(7239):780-783.

6. Wang T, Tamae D, LeBon T, Shively JE, Yen Y, Li JJ. The role of peroxiredoxin II in radiation-resistant MCF-7 breast cancer cells. Cancer Res. 2005;65(22):10338-10346.

7. Kowaltowski AJ, Netto LE, Vercesi AE. The thiol-specific antioxidant enzyme prevents mitochondrial permeability transition. Evidence for the participation of reactive oxygen species in this mechanism. $J$ Biol Chem. 1998;273(21):12766-12769.

8. Kowaltowski AJ, Vercesi AE, Rhee SG, Netto LE. Catalases and thioredoxin peroxidase protect Saccharomyces cerevisiae against $\mathrm{Ca}(2+)$-induced mitochondrial membrane permeabilization and cell death. FEBS Lett. 2000;473(2):177-182.

9. Netto LE, Kowaltowski AJ, Castilho RF, Vercesi AE. Thiol enzymes protecting mitochondria against oxidative damage. Methods Enzymol. 2002;348:260-270.

10. Rhee SG, Woo HA, Kil IS, Bae SH. Peroxiredoxin functions as a peroxidase and a regulator and sensor of local peroxides. J Biol Chem. 2012;287(7):4403-4410.

11. Netto LES, Chae HZ, Kang SW, Rhee SG, Stadtman ER. Removal of hydrogen peroxide by thiol-specific antioxidant enzyme (TSA) is involved with its antioxidant properties. TSA possesses thiol peroxidase activity. J Biol Chem. 1996;271(26):15315-15321.

12. Butterfield LH, Merino A, Golub SH, Shau H. From cytoprotection to tumor suppression: the multifactorial role of peroxiredoxins. Antioxid Redox Signal. 1999;1(4):385-402.

13. Biswas S, Chida AS, Rahman I. Redox modifications of protein-thiols: emerging roles in cell signaling. Biochem Pharmacol. 2006;71(5): 551-564.

14. Shau H, Kim AT, Hedrick CC, et al. Endogenous natural killer enhancing factor-B increases cellular resistance to oxidative stresses. Free Radic Biol Med. 1997;22(3):497-507.

15. Kim H, Lee TH, Park ES, et al. Role of peroxiredoxins in regulating intracellular hydrogen peroxide and hydrogen peroxide-induced apoptosis in thyroid cells. J Biol Chem. 2000;275(24):18266-18270.

16. Chung YM, Yoo YD, Park JK, Kim YT, Kim HJ. Increased expression of peroxiredoxin II confers resistance to cisplatin. Anticancer Res. 2001;21(2A):1129-1133.
17. Sarafian TA, Rajper N, Grigorian B, Kim A, Shau H. Cellular antioxidant properties of human natural killer enhancing factor B. Free Radic Res. 1997;26(3):281-289.

18. Wang T, Hu Y, Dong S, et al. Co-activation of ERK, NF-kappaB, and GADD45beta in response to ionizing radiation. J Biol Chem. 2005;280(13):12593-12601.

19. Wood ZA, Poole LB, Karplus PA. Peroxiredoxin evolution and the regulation of hydrogen peroxide signaling. Science. 2003;300(5619): 650-653.

20. Lushchak VI. Glutathione homeostasis and functions: potential targets for medical interventions. J Amino Acids. 2012;2012:736837.

21. Wood ZA, Schröder E, Robin Harris J, Poole LB. Structure, mechanism and regulation of peroxiredoxins. Trends Biochem Sci. 2003;28(1): 32-40.

22. Zheng M, Aslund F, Storz G. Activation of the OxyR transcription factor by reversible disulfide bond formation. Science. 1998;279(13): 1718-1721.

23. Chakraborty C. Potentiality of small interfering RNAs (siRNA) as recent therapeutic targets for gene-silencing. Curr Drug Targets. 2007;8(3): 469-482.

24. Mittal V. Improving the efficiency of RNA interference in mammals. Nat Rev Genet. 2004;5(5):355-365.

25. Whalley K. Breakthrough for systemic RNAi. Nat Rev Drug Dis. 2006;5:373.

26. Di Monte D, Bellomo G, Thor H, Nicotera P, Orrenius S. Menadioneinduced cytotoxicity is associated with protein thiol oxidation and alteration in intracellular $\mathrm{Ca} 2+$ homeostasis. Arch Biochem Biophys. 1984;235(2):343-350.

27. Bellomo G, Nicotera P, Orrenius S. Alterations in intracellular calcium compartmentation following inhibition of calcium efflux from isolated hepatocytes. Eur J Biochem. 1984;144(1):19-23.

28. Duru N, Fan M, Candas D. Her2-associated Radioresistance of Breast Cancer Stem Cells Isolated from Her2-Negative Breast Cancer Cells. Clin Cancer Res. 2012,18:6634-6647.

29. Valent P, Bonnet D, De Maria R, et al. Cancer stem cell definitions and terminology: the devil is in the details. Nat Rev Cancer. 2012;12(11): 707-775.

30. Park S, Chung YM, Lee YS, et al. Antisense of human peroxiredoxin II enhances radiation-induced cell death. Clin Cancer Res. 2000;6(12): 4915-4920.

31. Whalley K. Breakthrough for systemic RNAi. Nat Rev Drug Dis. 2006;5:373.

32. Bellomo G, Jewell SA, Thor H, Orrenius S. Regulation of intracellular calcium compartmentation: studies with isolated hepatocytes and t-butyl hydroperoxide. Proc Natl Acad Sci U S A. 1982;79(22):6842-6846.

33. Sen CK. Cellular thiols and redox-regulated signal transduction. Curr Top Cell Regul. 2000;36:1-30.

34. Clapham DE. Calcium signaling. Cell. 2007;131:1047-1058.

Breast Cancer: Targets and Therapy

\section{Publish your work in this journal}

Breast Cancer: Targets and Therapy is an international, peerreviewed open access journal focusing on breast cancer research, identification of therapeutic targets and the optimal use of preventative and integrated treatment interventions to achieve improved outcomes, enhanced survival and quality of life for the cancer patient. 